\title{
La corruption du composé de l'âme et du corps selon Descartes
}

\section{Géraldine Caps}

\section{(2) OpenEdition \\ 1 Journals}

Édition électronique

URL : http://journals.openedition.org/edl/923

DOI : $10.4000 /$ edl. 923

ISSN : 2296-5084

Éditeur

Université de Lausanne

\section{Édition imprimée}

Date de publication : 15 décembre 2015

ISBN : 978-2-940331-47-5

ISSN : 0014-2026

\section{Référence électronique}

Géraldine Caps, "La corruption du composé de l'âme et du corps selon Descartes 》, Études de lettres [En ligne], 3-4 | 2015, mis en ligne le 01 décembre 2018, consulté le 16 décembre 2020. URL : http:// journals.openedition.org/edl/923 ; DOI : https://doi.org/10.4000/edl.923

\section{(C) Études de lettres}




\section{LA CORRUPTION DU COMPOSÉ DE LÂME ET DU CORPS SELON DESCARTES}

Bien que le terme "corruption» soit rarement employé par Descartes, ses occurrences s'avèrent en parfaite conformité avec l'ensemble des thèses constitutives de son système. Alors que l'acception du terme varie selon ce à quoi il se rapporte, il n'en reste pas moins que ses usages se rencontrent à des moments philosophiques charnières. Ainsi, loin d'être reléguée au second plan, la notion de corruption revêt de toute évidence une importance qui, jusqu'alors, a été largement sous-estimée, voire occultée. Dans le dessein de contrer la tradition scolastique, Descartes insiste sur l'objectif fondamental de son œuvre philosophique, à savoir la nécessité d'atteindre une connaissance claire et distincte dans les sciences. Cette lecture épistémologique se double d'une réflexion philosophique, anthropologique et morale sur le travail qu'un individu entreprendra sur ses propres représentations aux seules fins de ruiner la forme de corruption que sont les préjugés et les utopies qu'il véhicule, souvent depuis l'enfance, aussi bien sur certains phénomènes du monde qui l'entourent que sur lui-même.

Chez Descartes, la notion de corruption, entendue au sens de corruption du tout qu'est l'homme, occupe une place cardinale dans l'étude du cas pathologique de l'hydropique. Le philosophe peut alors approfondir sa réflexion anthropologique sur l'union de l'âme et du corps, tout en ouvrant de nouvelles perspectives épistémologiques et pratiques en médecine. Toutefois, ce point s'avère paradoxal, et constitue quasiment un cas limite. En effet, la troisième des «notions primitives» ${ }^{1}$ présente

I. "J'ai distingué trois genres d'idées ou de notions primitiues qui se connoissent chacune d'vne façon particuliere $\&$ non par la comparaison de l'vne à l'autre, à sçauoir la notion que nous auons de l'ame, celle du corps, \& celle de l'vnion qui est entre l'ame \& le corps» (Lettre à Elisabeth, 28 juin 1643, A.T. III, p. 691). 
un trait particulier en ce qu'elle est à la fois unitaire, mais également composée de deux entités hétérogènes qui sont elles-mêmes des «notions primitives» et qui ordinairement sont intimement liées, tout en disposant aussi simultanément d'une indépendance partielle réciproque; le corps en tant que substance étendue - pouvant agir indépendamment de l'âme - dont l'essence est d'être une substance pensante - et réciproquement. Néanmoins, un cas pathologique comme l'hydropisie va porter atteinte à l'union de l'âme et du corps, sans aller jusqu'à la mettre radicalement en péril comme le ferait une destruction totale; ce qui constitue une différence de degré entre le cas pathologique et la mort. Par corruption, il faut donc ici entendre, une altération de l'union substantielle de l'âme et du corps par sa mise en porte à faux, laquelle mise en porte à faux devient radicale et devient une dissociation dans la décomposition totale de l'union de l'âme et du corps qu'est la mort.

L'analyse du cas de l'hydropique sous l'angle de la corruption de l'âme et du corps laisse apparaître plusieurs enjeux: qu'en est-il de l'identité de l'individu? Comment penser une certaine permanence identitaire - le sujet est toujours identifié comme tel - et les changements pathologiques qui affectent et qui modifient provisoirement ou durablement son corps? Quels sont les enjeux et les conséquences d'un point de vue thérapeutique, philosophique, métaphysique, anthropologique? Nous voudrions dans un premier temps analyser le cas pathologique de la corruption du composé en rapport avec l'hydropisie, pour aborder dans un second temps les questions de la finitude et de l'identité qu'il soulève.

\section{L'hydropique ou une corruption partielle pathologique du composé}

Bien que Descartes insiste sur l'absolue hétérogénéité des substances pensante et corporelle, ces deux substances forment néanmoins, via la troisième des "notions primitives", le tout qu'est l'homme. L'intime union de l'âme et du corps présente paradoxalement une corruption de nature pathologique:

Nous nous trompons aussi assez souuent, mesme dans les choses ausquelles nous sommes directement portez par la nature, comme il arriue aux malades, lorsqu'ils desirent de boire ou de manger des choses qui leur peuuent nuire. On dira peut-estre icy que ce qui est 
cause qu'ils se trompent, est que leur nature est corrompuë; mais cela n'oste pas la difficulté, parce qu'vn homme malade n'est pas moins veritablement la creature de Dieu, qu'vn homme qui est en pleine santé; $\&$ partant il repugne autant à la bonté de Dieu, qu'il ait vne nature trompeuse \& fautiue, que l'autre. Et comme vne horloge, composée de roües $\&$ de contrepoids, n'obserue pas moins exactement toutes les loix de la nature, lorsqu'elle est mal faite, \& qu'elle ne montre pas bien les heures, que lorsqu'elle satisfait entierement au desir de l'ouurier; de mesme aussi, si ie considere le corps de l'homme comme estant vne machine tellement bastie \& composée d'os, de nerfs, de muscles, de veines, de sang $\&$ de peau, qu'encore bien qu'il n'y eust en luy aucun esprit, il ne lairroit pas de se mouuoir en toutes les mesmes façons qu'il fait à present, lorsqu'il ne se meut point par la direction de sa volonté, ny par consequent par l'aide de l'esprit, mais seulement par la disposition de ses organes, ie reconnois facilement qu'il seroit aussi naturel à ce corps, estant, par exemple, hydropique, de souffrir la secheresse du gozier, qui a coustume de signifier à l'esprit le sentiment de la soif, $\&$ d'estre disposé par cette secheresse à mouuoir ses nerfs $\&$ ses autres parties, en la façon qui est requise pour boire, $\&$ ainsi d'augmenter son mal $\&$ se nuire à soy-mesme, qu'il luy est naturel, lorsqu'il n'a aucune indisposition, d'estre porté à boire pour son vtilité par vne semblable secheresse de gozier ${ }^{2}$.

Le cas pathologique de l'hydropique comporte moins une dimension polémique qu'il ne vise à mettre en évidence la pertinence et la validité du système que défend Descartes. En effet, alors que la tradition médicale reste en échec pour rendre compte de cette pathologie (elle la conçoit comme échappant à l'ordre et aux lois de la nature), Descartes dépasse l'aporie où en est restée la tradition. Comme le souligne Annie Bitbol-Hespériès, "l'analyse cartésienne met à mal l'invocation du thème de la nature dans les traités médicaux, et les distinctions qui en sont issues, classant l'hydropisie parmi les tumeurs contre nature» ${ }^{3}$. Selon Descartes, l'hydropique présente une nature corrompue et par là pathologique, mais il n'en reste pas moins que la pathologie obéit encore aux lois physiques de la nature. Même atteint dans son fonctionnement normal, le corps humain appartient encore à l'ordre de la nature et reste une création divine gardant son identité:

2. Ibid., A.T. IX-1, p. 67.

3. A. Bitbol-Hespériès, "La médecine et l'union dans la Méditation sixième», p. 35. 
Mais certes, quoy qu'au regard du corps hydropique, ce ne soit qu'vne denomination exterieure, lors qu'on dit que sa nature est corrompuë, en ce que, sans auoir besoin de boire, il ne laisse pas d'auoir le gozier sec $\&$ aride; toutesfois, au regard de tout le composé, c'est à dire de l'esprit ou de l'ame vnie à ce corps, ce n'est pas vne pure denomination, mais bien vne veritable erreur de nature, en ce qu'il a soif, lorsqu'il luy est tres-nuisible de boire; \& partant, il reste encore à examiner comment la bonté de Dieu n'empesche pas que la nature de l'homme, prise de cette sorte, soit fautiue $\&$ trompeuse ${ }^{4}$.

Cette «veritable erreur de la nature» que donne à voir l'hydropisie est à distinguer d'abord de la corruption totale du corps qu'est la mort, mais aussi de la variabilité naturelle du corps, et enfin de la dégradation d'une partie du corps, exigeant une amputation. Il convient de préciser que la variabilité naturelle du corps appartient à l'essence de ce dernier et est particulièrement manifeste lorsque le corps grandit, grossit, vieillit, se régénère et / ou se transforme selon un processus naturel. Ces changements incessants font que l'unité, l'unicité et l'identité de l'homme ne sont pas à attribuer à son corps, mais à rapporter à l'âme; ce qui implique que le corps d'un individu est proprement le sien en raison de l'âme qui lui est unie:

Je ne pense pas qu'il y ait aucune particule de nos membres, qui demeure la mesme numero vn seul moment, encore que nostre corps, en tant que corps humain, demeure tousiours le mesme numero, pendant qu'il est vny auec la mesme ame. Et mesme, en ce sens là, il est indiuisible: car, si on coupe vn bras ou vne jambe a vn homme, nous pensons bien que son corps est diuisé, en prenant le nom de corps en la ${ }^{\text {re }}$ signification, mais non pas en la prenant en la $2^{\text {de }}$; et nous ne pensons pas que celuy qui a vn bras ou vne iambe couppée, soit moins homme qu'vn autre. Enfin, quelque matiere que ce soit, $\&$ de quelque quantité ou figure qu'elle puisse estre, pourueu qu'elle soit vnie auec la mesme ame raisonnable, nous la prenons tousiours pour le corps $\mathrm{du}$ mesme homme, \& pour le corps tout entier 5 .

Même si les deux substances que sont l'âme et le corps sont unies, elles gardent une indépendance relative et l'âme reste, quoi qu'il arrive au corps, d'une indivisibilité absolue: de fait, la corruption du corps

4. Méditations, A.T. IX-1, p. 68.

5. Lettre au Père Mesland, 9 février 1645, A.T. IV, p. 167. 
n'entraîne pas la corruption de l'âme ${ }^{6}$. Les cas de gangrène ou d'autres pathologies qui amènent à la prescription d'une amputation permettent philosophiquement de préciser les attributs de la substance étendue. Non seulement cette dernière possède une certaine variabilité naturelle, mais également, à la différence de la substance pensante, une divisibilité qui est partie intégrante de son essence:

Le corps, de sa nature, est tousiours diuisible, \& [...] l'esprit est entierement indiuisible. Car en effect, lors que ie considere mon esprit, c'est à dire moy-mesme en tant que ie suis seulement vne chose qui pense, ie n'y puis distinguer aucunes parties, mais ie me conçoy comme vne chose seule \& entiere. Et quoy que tout l'esprit semble estre vny à tout le corps, toutesfois vn pied, ou vn bras, ou quelqu'autre partie estant séparée de mon corps, il est certain que pour cela il n'y aura rien de retranché de mon esprit ${ }^{7}$.

Par essence, le corps admet donc une certaine divisibilité, ce qui fait que l'identité - au sens d'une permanence individuelle - ne peut en soi résider dans le corps. Seule l'âme marque l'identité pour Descartes, car elle s'avère indivisible et in fine inaltérable. Par son union avec le corps, l'âme fait qu'il devient un corps proprement humain; ce qui implique des conséquences dans la façon de considérer le corps sain ou pathologique et engage des enjeux liés à la façon d'appréhender l'être humain tout entier. Nous y reviendrons.

$\mathrm{Du}$ point de vue médical et thérapeutique, les bornes du fonctionnement du corps sont mises en évidence, ce qui signifie qu'il demeure nécessairement (et par essence) une imprécision relative aux mécanismes du corps et que cette imprécision est parfois, mais pas nécessairement, source de maladies. Le mécanisme provoquant la sensation de soif fonctionne indépendamment des besoins effectifs du corps au sein duquel il advient, ce qui fait que la soif sera suscitée, même si elle est nuisible, comme c'est le cas pour l'hydropique. Cette indécision se rapporte indiscutablement à la nature finie de l'homme et à l'absence

6. "De la corruption du corps la mort de l'ame ne s'ensuit pas", Abregé des six meditations svivantes, A.T. IX-1, p. 10.

7. Méditations, A.T. IX-1, p. 68. 
de finalité interne au mécanisme ${ }^{8}$. Par voie de conséquence, la médecine - que ce soit dans son épistémologie, dans sa partie théorique, dans son exercice et même dans sa réflexion déontologique sur le devoir d'état, les possibilités et les limites du médecin - ne peut ni nier, ni dépasser l'imprécision de l'objet qu'elle traite et n'a d'autre choix que d'intégrer cette limite irréductible, laquelle devient une borne à une scientificité mathématique absolue. De fait, l'espace ouvert par l'imprécision relative au corps humain, invite à accepter et à assumer la finitude constitutive de la nature humaine dans l'agir - collectif et / ou individuel - médical et thérapeutique, ainsi quau quotidien.

\section{L'identité et la finitude humaines mises en question}

La question de l'identité humaine est posée en vertu de l'imprécision qu'entretient le cas de l'hydropique au niveau de l'union de l'âme et du corps, lequel n'est pas exactement identique - sans néanmoins y être toutefois complètement étranger - au cas de l'amputé, vu que dans ce dernier cas un segment corporel a été enlevé, alors que l'hydropique dispose d'un corps a priori entier, mais qui s'avère néanmoins disfonctionnel. Quelles sont donc les conditions d'existence et de persistance pour penser l'identité d'une personne? Jusqu'où peut-il y avoir changement, altération et/ou modération tantôt du corps, tantôt de l'union de l'âme et du corps sans néanmoins porter irrémédiablement atteinte à l'identité de la personne? La perte d'une unité identitaire interne présente-t-elle nécessairement des conséquences vitales?

Nous disons que nous voyons la mesme cire, si on nous la presente, $\&$ non pas que nous iugeons que c'est la mesme, de ce qu'elle a mesme couleur $\&$ mesme figure: d'où ie voudrois presque conclure, que l'on connoist la cire par la vision des yeux, $\&$ non par la seule inspection

8. Le cas de l'hydropique "ouvre la possibilité d'un mécanisme aveugle et d'une finalité impuissante. Les plans, jusque-là confondus, du mécanisme et de la finalité, se décollent l'un de l'autre et ce décrochage suscite le conflit dont l'hydropique est l'occasion ou l'exemple. Car ce n'est pas au mécanisme que l'hydropique porte atteinte: 'ça' fonctionne toujours de la même façon, mais plus dans le même sens, celui de l'utilité. On découvre avec l'hydropique l'indifférence de la nature, de la machine, du mécanisme à l'utile comme au nuisible» (P. Guenancia, Lire Descartes, p. 280). 
de l'esprit, si par hazard ie ne regardois d'vne fenestre des hommes qui passent dans la ruë, à la veuë desquels ie ne manque pas de dire que ie voy des hommes, tout de mesme que ie dis que ie voy de la cire; Et cependant que voy-je de cette fenestre, sinon des chapeaux \& des manteaux, qui peuuent couurir des spectres ou des hommes feints qui ne se remuent que par ressors? Mais ie iuge que ce sont de vrais hommes; \& ainsi ie le comprens, par la seule puissance de iuger qui reside en mon esprit, ce que ie croyois voir de mes yeux ${ }^{9}$.

Le jugement engage le regard que porte un individu sur un autre, à savoir, dans le cas présent, le regard d'un médecin sur un malade ou encore le regard du malade sur lui-même. Le jugement doit donc être suffisamment affiné pour être apte à distinguer la maladie du malade, ce qui revient à ne pas assimiler l'identité du malade à la maladie. Néanmoins, sans en rester à de seules apparences physiques figées, l'identité implique un minimum de ressemblance. L'identité n'est pas alors synonyme d'une permanence absolue d'un état initial, mais elle paraît pourtant impliquer, au moins physiquement:

[...] une resemblance des dimensions: comme nous pouuons dire que la Loire est la mesme riuiere qui estoit il y a dix ans, bien que ce ne soit plus la mesme eau, \& que peut estre aussi il n'y ait plus aucune partie de la mesme terre qui enuironnoit cette eau ${ }^{10}$.

Toutefois, ce critère de ressemblance qui s'applique aux corps étudiés par la physique et par la philosophie naturelle, demeure insuffisant dans la considération du corps humain, ce qui amène Descartes à développer spécifiquement une anthropologie:

Quand nous parlons du corps d'vn homme, nous n'entendons pas vne partie determinée de matiere, ny qui ait vne grandeur determinée, mais seulement nous entendons toute la matiere qui est ensemble vnie auec l'ame de cet homme; en sorte que, bien que cette matiere change, \& que sa quantité augmente ou diminuë, nous croyons tousiours que c'est le mesme corps, idem numero, pendant qu'il demeure ioint \& vny substantiellement a la mesme ame; $\&$ nous croyons que ce corps est

9. Méditations, A.T. IX-1, p. 25.

Io. Lettre au Pere Mesland, 9 février 1645, A.T. IV, p. 165. 
tout entier, pendant qu'il a en soy toutes les dispositions requises pour conseruer cette vnion ${ }^{11}$.

Descartes utilise à plusieurs reprises le verbe «croire», ce qui marque un changement de registre dans l'ordre de la connaissance: il sort de l'ordre des raisons théorétiques, d'une connaissance issue de la raison spéculative démonstrative au profit d'une connaissance plus empirique. La Lettre à Mesland donne encore dans le registre de la croyance ${ }^{12}$, ce qui montre bien les difficultés à connaître spéculativement l'identité de l'homme, alors que dans la vie quotidienne - où la connaissance pratique et le bon sens fournissent des critères d'action -, nous reconnaissons sans grande difficulté nos congénères. Ainsi l'altération, au sens de corruption partielle, du corps humain fait-elle davantage problème dans la conduite d'une réflexion philosophique que dans la vie pratique. Il demeure néanmoins que Descartes ne pose pas la question du seuil: comment peut-on déterminer les limites des modifications (volontaires ou non) corporellement imposables et / ou imposées, soit pour reconnaître et identifier un individu, soit pour ne pas tomber en deçà d'un seuil vital?

L'erreur de la nature qui constitue le cas de l'hydropique renvoie éminemment à la finitude humaine. Le rapport que l'individu entretient avec cette finitude peut être interrogé : faut-il la subir au même titre qu'une fatalité ou faut-il l'accepter comme naturelle et comme constitutive de son essence? Descartes accepte la finitude humaine, ainsi que cela paraît particulièrement dans la troisième maxime qu'il se donne dans le Discours de la méthode, où la corruption du corps est posée comme étant partie intégrante de la finitude de l'homme et de son essence:

Ma troisieme maxime estoit de tascher tousiours plutost a me vaincre que la fortune, $\&$ a changer mes desirs que l'ordre du monde; \& generalement, de m'accoustumer a croire qu'il n'y a rien qui soit entierement en nostre pouuoir, que nos pensées, en sorte qu’aprés

II. Ibid., A.T. IV, p. 166.

I2. "Car il n'y a personne qui ne croye que nous auons les mesmes corps que nous auons eus des nostre enfance, bien que leur quantité soit de beaucoup augmentée, \& que, selon l'opinion commune des Medecins, \& sans doute selon la verité, il n'y ait plus en eux aucune partie de la matiere qui y estoit alors, \& mesme qu'ils n'ayent plus la mesme figure; en sorte qu'ils ne sont eadem numero, qu'a cause qu'ils sont informez de la mesme ame» (ibid., p. 166 sq.). 
que nous auons fait nostre mieux, touchant les choses qui nous sont exterieures, tout ce qui manque de nous reussir est, au regard de nous, absolument impossible. Et cecy seul me sembloit estre suffisant pour m'empescher de rien desirer a l'auenir que ie n'acquisse, $\&$ ainsi pour me rendre content. Car nostre volonté ne se portant naturellement a desirer que les choses que nostre entendement luy represente en quelque façon comme possibles, il est certain que, si nous considerons tous les biens qui sont hors de nous comme esgalement esloignez de nostre pouuoir, nous n'aurons pas plus de regret de manquer de ceux qui semblent estre deus a nostre naissance, lorsque nous en serons priuez sans nostre faute, que nous auons de ne posseder pas les royaumes de la Chine ou de Mexique; \& que faisant, comme on dit, de necessité vertu, nous ne desirerons pas dauantage d'estre sains, estant malades, ou d'estre libres, estant en prison, que nous faisons maintenant d'auoir des cors d'vne matiere aussy peu corruptible que les diamans, ou des ailes pour voler comme les oiseaux. Mais i'auouë qu'il est besoin d'vn long exercice, \& d'vne meditation souuent reïterée, pour s'accoustumer a regarder de ce biais toutes les choses ${ }^{13}$.

Ainsi Descartes bat-il en brèche le phantasme de l'immortalité du corps: même si le corps peut être assimilé à une machine et, ipso facto, être partie prenante d'une représentation techniciste et technicienne du monde, il n'en reste pas moins qu'il demeure faillible et qu'il est impossible de le «réparer» à l’infini.

Par ailleurs, la réflexion concernant la finitude humaine est soustendue par la topique de la corruption des mœurs, évoquée dans Le Discours de la méthode ${ }^{14}$ et dont on sait qu'elle comporte une forte dimension polémique. Descartes entend en effet combattre les écrits hétérodoxes de son temps, prompts à peindre l'impiété et à promouvoir le vice. Aux arguties des libertins, Descartes oppose l'immatérialité et l'immortalité de l'âme (il définit la mort comme la corruption du corps en tant que substance étendue ${ }^{15}$ ). Les enjeux de la corruption des mœurs

13. Discours de la méthode, A.T. VI, p. 25 sq.

I4. «[...] non seulement a cause qu'en la corruption de nos mœurs il y a peu de gens qui veuillent dire tout ce qu'ils croyent, mais aussy a cause que plusieurs l'ignorent eux mesmes; car l'action de la pensée par laquelle on croit vne chose, estant differente de celle par laquelle on connoist qu'on la croit, elles sont souuent l'vne sans l'autre» (Discours de la méthode, A.T. VI, p. 22 sq.).

I5. "L'ame ne s'absente lors qu'on meurt, qu'à cause que cette chaleur [corporelle] cesse, \& que les organes qui servent à mouvoir le corps se corrompent. [...] La mort 
vont bien au-delà du simple libertinage. En effet, la pensée de Descartes met l'accent sur la difficulté qu'il y a d'une part à connaître en général, et, d'autre part, à se connaître soi-même. Ainsi Descartes fait-il allusion, en creux, à sa méthode de connaissance, qui exige, "vne fois en [s]a vie" ${ }^{16}$, de faire porter le doute sur tout, y compris sur soi-même, et de trouver, au moyen de ce doute radical et hyperbolique, les fondements de toute connaissance vérace. Aussi, la corruption des mœurs renvoie-telle à un constat anthropologique et phénoménologique propre à mettre en lumière la nature humaine et l'essence de l'agir humain. Cette corruption, ou cette tendance à la corruption d'ordre pratique, reflète la finitude de l'homme et s'avère en être constitutive, ce dont le langage porte la trace. À Marin Mersenne qui évoque le projet d'une langue universelle, Descartes répond:

Faisant vne langue, où il n'y ait qu'vne façon de conjuguer, de decliner, $\&$ de construire les mots, qu'il n'y en ait point de defectifs ny d'irriguliers, qui sont toutes choses venuës de la corruption de l'vsage, [...] ce ne sera pas merueille que les esprits vulgaires apprennent en moins de six heures à composer en cette langue ${ }^{17}$.

Cette corruption de l'usage, qui fait, sous un certain angle, écho à la corruption des mœurs, renvoie elle-même et de façon quasi tautologique à un constat et une connaissance anthropologiques: la finitude de l'homme, laquelle constitue un support favorable au développement de la corruption de l'usage. La finitude de l'homme - dont Descartes tient lucidement compte, mais sans néanmoins donner dans un pessimisme exacerbé - demeure partie intégrante aussi bien des actions humaines que de son être même.

\section{Une anthropologie humaine renforcée}

L'existence semble impliquer une permanence de l'essence en tant qu'espèce et en tant qu'être singulier. L'identité paraît en effet ne pas être

n'arrive jamais par la faute de l'ame, mais seulement parce que quelcune des principales parties du corps se corrompt» (Passions de l'âme, A.T. XI-1, p. 330).

16. Méditations, A.T. IX-1, p. 13.

17. Lettre à Marin Mersenne, 20 novembre 1629, A.T. I, p. 77. 
seulement une relation à soi, mais présente une implication indéfectible en soi qui trouve son siège et son état dans l'âme humaine, d'où une persistance identitaire tout au long de la vie. En dépit des variations et de la variabilité corporelles naturelles, l'identité corporelle et l'identité de la personne se trouvent et se retrouvent en et par l'âme, ce qui fait que Descartes insiste fortement sur le rôle de l'âme, particulièrement dans Les passions de l'âme:

L'ame est veritablement jointe à tout le corps, \& [...] on ne peut pas proprement dire qu'elle soit en quelcune de ses parties, à l'exclusion des autres, à cause qu'il est un, $\&$ en quelque façon indivisible, à raison de la disposition de ses organes, qui se raportent tellement tous l'un à l'autre, que lors que quelcun d'eux est osté, cela rend tout le corps defectueux; \& à cause qu'elle est d'une nature qui n'a aucun raport à l'estendue, ny aux dimensions, ou autres proprietez de la matiere dont le corps est composé, mais seulement à tout l'assemblage de ses organes. Comme il paroist, de ce qu'on ne sçauroit aucunement concevoir la moitié ou le tiers d'une ame, ny quelle estendue elle occupe, $\&$ qu'elle ne devient point plus petite de ce qu'on retranche quelque partie du corps, mais qu'elle s'en separe entierement, lors qu'on dissout l'assemblage de ses organes ${ }^{18}$.

La tension qui pouvait être générée eu égard au statut de l'âme par rapport à la divisibilité du corps humain, tombe au nom de l'indivisibilité et de l'indéfectibilité de l'âme. L'identité humaine ne se réduit donc pas à un état corporel, ni à une fonction. De ce point de vue, l'âme est si importante pour Descartes qu'il estime que "c'est moins de perdre la vie que de perdre l'vsage de la raison " ${ }^{19}$. Ainsi, au nom de la synonymie que Descartes pose entre mens et anima, pouvons-nous considérer cette accentuation de la place suprême de l'âme dans sa réflexion comme une mise en exergue de la spécificité de l'identité humaine. L'âme est à la fois unificatrice de l'être humain mais également l'instrument du bonheur:

Vous remarquez fort bien qu'il y a des maladies qui, ostant le pouuoir de raisonner, ostent aussy celuy de iouir d'vne satisfaction d'esprit raisonnable; \& cela m'apprent que ce que i'auois dit generalement de tous les hommes, ne doit estre entendu que de ceux qui ont l'vsage libre de leur raison, \& auec cela qui sçauent le chemin qu'il faut tenir pour

18. Passions de l'âme, A.T. XI, p. 351.

19. Lettre à Elisabeth, ${ }^{\mathrm{er}}$ septembre 1645, A.T. IV, p. 282. 
paruenir a cete beatitude. Car il n'y a personne qui ne desire se rendre hureux; mais plusieurs n'en sçauent pas le moyen; \& souuent l'indisposition qui est dans le corps, empesche que la volonté ne soit libre. Comme il arriue aussy quand nous dormons; car le plus philosophe du monde ne sçauroit s'empescher d'auoir de mauuais songes, lorsque son temperament l'y dispose. Toutefois l'experience fait voir que, si on a eu souuent quelque pensée, pendant qu'on a eu l'esprit en liberté, elle reuient encore apres, quelque indisposition qu'ait le cors; ainsy ie puis dire que mes songes ne me representent iamais rien de fascheux, \& sans doute qu'on a grand auantage de s'estre des long tems accoustumé a n'auoir point de tristes pensées. Mais nous ne pouuons respondre absolument de nous mesmes, que pendant que nous sommes a nous, $\&$ c'est moins de perdre la vie que de perdre l'vsage de la raison; car, mesme sans les enseignemens de la foy, la seule philosophie naturelle fait esperer a nostre ame vn estat plus heureux, apres la mort, que celuy ou elle est a present; \& elle ne luy fait rien craindre de plus fascheux, que d'estre attachée a vn cors qui luy oste entierement sa liberté ${ }^{20}$.

L'anthropologie philosophique de Descartes ouvre, on le voit, à des obligations thérapeutiques, au nom de l'union de l'âme et de corps et de l'humanité inhérente au corps humain. Le soin de tout dérèglement somatique devra aussi essayer d'en minimiser les conséquences sur l'âme, et ainsi veiller à préserver, autant que faire se peut, la liberté et la possibilité du contentement pour chaque individu. Alors que le corps humain est marqué par la maladie et par la corruption, cet état de fait constitue une occasion pour Descartes de clarifier et d'approfondir son système philosophique, et particulièrement son anthropologie. Un regard humain affiné et la suprématie de l'âme deviennent des moyens philosophiques de dépasser la corruption naturelle du corps humain et de transcender le temps qui passe.

Géraldine CAPS

Université de Montréal

20. Ibid., p. 281 sq. 


\section{BIBLIOGRAPHIE}

Textes

Descartes, René, Euvres de Descartes (A.T.), éd. par Charles Adam et Paul Tannery, Paris, Vrin/CNRS, 1964-1976.

—, Règles pour la direction de l'esprit, éd. par Jean Sirven, Paris, Vrin, 1996.

\section{Travaux}

Bitbol-Hespériès, Annie, «La médecine et l'union dans la Méditation sixième", in Union et distinction de l'âme et du corps: lectures de la $V I^{\mathrm{e}}$ méditation, éd. par Delphine Kolesnik-Antoine, Paris, Kimé, 1998, p. 18-36.

Guenancia, Pierre, Lire Descartes, Paris, Gallimard, 2000. 
